\title{
Efficacy of Transumbilical single incision-Laparoscopic- assisted surgery in pediatric patients
}

\author{
Soo-Min Jung* \\ Department of Surgery, Konkuk University Medical Center, Konkuk University, School of Medicine, Seoul, Korea
}

\begin{abstract}
Backgrounds: Due to advancement in minimally invasive surgery, the boundary of single incision laparoscopic surgery is extended to pediatric patients. We investigated the usefulness of transumbilical single incision laparoscopic- assisted surgery based on our recent experiences.

Methods: We retrospectively collected the data of 82 patients who underwent transumbilical single incision laparoscopic- assisted surgery from May 2013 to January 2016

Results: During the study period, 82 children underwent surgery. 72 (87.8\%) patients underwent appendectomy with TSI and the remaining 10 patients underwent transumbilical single incision laparoscopic- assisted surgery for various surgical abdominal conditions such as intussusception, Meckel's diverticulum, small bowel mass, mesenteric lymphangioma, adhesive ileus, and the like. The Extracorporeal procedure was performed alongside with laparoscopy for sophisticated manipulation. Mean operation time was $40 \pm 20$ minutes in appendicitis while other operations were held for 30 to 80 minutes. The reduction of intussusception can be done easily and take a short operation time with transumbilical single incision laparoscopic- assisted surgery. Median hospital days was 3 days (2 7 days) in appendicitis while others stayed for 3 to 9 days. There was no surgical complication during admission. Three patients underwent appendectomy had umbilical stitch abscess a month later which resolved after removing the stitch.
\end{abstract}

Conclusion: We consider with transumbilical single incision laparoscopic- assisted surgery as a safe procedure in pediatric patients. And the umbilical window makes surgery easier. Even in challenging cases, exposure of the lesion through the umbilical port helps to avoid conversion to laparotomy.

\section{Introduction}

The innovation in minimally invasive surgeries, especially laparoscopy, has changed the paradigm of surgery. Since Pelosi, et al. first introduced single-incision laparoscopic appendectomy, single port surgery has been amended to many fields of surgery [1]. However, the limited space inside the abdomen of pediatric patients makes it difficult to perform the procedure, resulting in a delay of utilizing single port surgery. Nonetheless, some investigators reported performing transumbilical laparoscopic assisted appendectomy, using the umbilical port for extracorporeal procedure Yury Kozlov, et al. reviewed the indications for single-incision endoscopic surgery (SIPES) in neonates and children under 12 months of age [2]. However, in the pediatric surgical field, there are not many reports of extracorporeal procedures through the single incision navel window in other surgical procedures, besides appendectomy. As reported by previous investigators, the author initiated single incision endoscopic surgery for appendectomy; most of the cases with single incision surgery are solely appendectomy. Since the umbilical port site can be utilized as an open window to perform extracorporeal procedures, surgeries other than appendectomy are easier, compared to conventional multiport laparoscopic surgery. This paper describes the experience of one pediatric surgeon, and discusses the efficacy of this procedure.

\section{Materials and methods}

This study was approved by the Institutional Review Board (\#2016-05-031). It included 82 pediatric patients who underwent transumbilical single incision laparoscopic-assisted surgery between May 2013 to January 2016, performed by a single pediatric surgeon.
The age of the patient, diagnosis, hospitalization period, operation time, and postoperative complications were retrospectively reviewed. The surgical procedure was as follows: a median or S-shape incision with a length of 1.5 2 cm was made on the umbilicus (Figure 1A). Through the incision, Glove port ${ }^{\mathrm{TM}}$ with a detachable cap was inserted (Figure 1B) A $5 \mathrm{~mm}$ camera and two $3 \mathrm{~mm}$ instruments were inserted through the port, and a $5 \mathrm{~mm}$ instrument and a $5 \mathrm{~mm}$ harmonic scalpel were used as energy devices, if required (Figure 1C). Occasionally, the cap of the Glove port ${ }^{\mathrm{TM}}$ was detached and extracorporeal manual procedure was performed through the port site. For example, intracorporeal laparoscopic appendicular artery ligation and appendix base ligation were performed for appendectomy, and the appendix was removed via the navel window following excision. During transumbilical single incision laparoscopic-assisted surgery, the extracorporeal procedure was performed through the umbilical port after the laparoscopic procedure, only when necessary. After completion of the manual procedure outside the abdomen, the small bowel was replaced within the abdominal cavity, following which the operation was concluded

Correspondence to: Soo-Min Jung, MD, Department of Surgery, Konkuk University Medical Center, Seoul, 05030, Korea, Tel: 82220307894, Fax: 82220307346, E-mail: lettoyam@naver.com

Key words: minimally invasive surgery, pediatric, laparoscopy, appendectomy, intussusception

Received: January 12, 2018; Accepted: February 05, 2018; Published: February 09,2018 


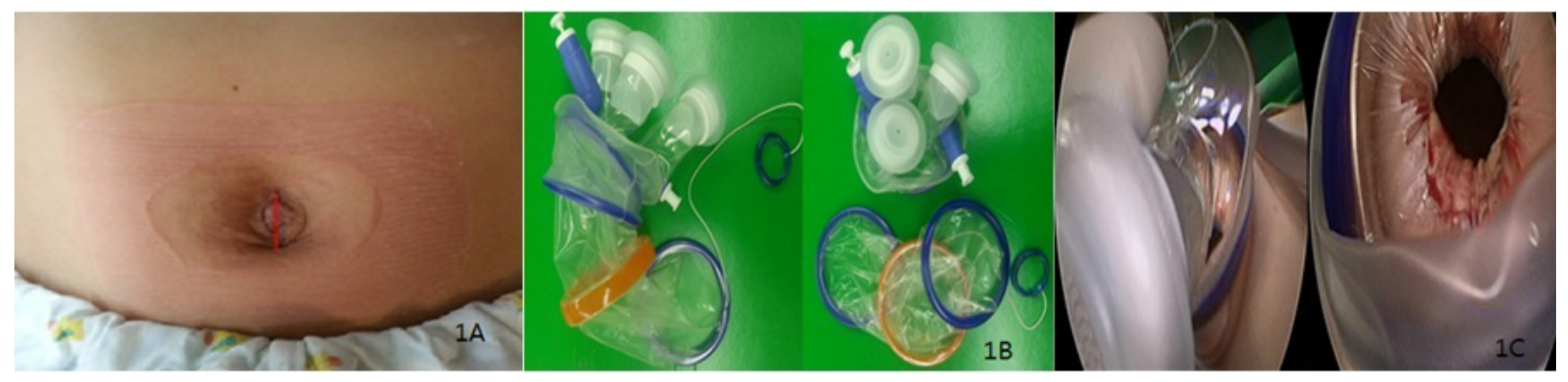

Figure 1. A) Incision site and shape, B) Cap-detachable Glove port $\left.{ }^{\mathrm{TM}}, \mathrm{C}\right)$ Glove port inserted through the umbilicus, and the appearance of the umbilicus

\section{Results}

The demographic features and diagnoses of the 82 patients are summarized in Table 1. The median age of the patients was 8.9 years, ranging from 4.3 months to 16.7 years. Seventy-two (87.8\%) patients underwent transumbilical single incision laparoscopic-assisted surgery for acute appendicitis (simple or micro perforation), which was the most common disorder. On the computed tomography, 1 patient was diagnosed with an incidental mass in the small bowel which was taken as a diagnosis of acute appendicitis. The patient underwent transumbilical single incision laparoscopic-assisted appendectomy, following which the terminal ileum was delivered through the umbilical port site and proximal bowel was traced manually. A $1 \mathrm{~cm}$ sized mass was found in the jejunum, and small bowel resection and anastomosis were performed extracorporeally. Pathology confirmed the mass as an ectopic pancreas. Transumbilical single incision laparoscopic-assisted surgery was performed on 6 patients with intussusception. The first patient had ileo-ileal intussusception as a complication of Henochschonlein purpura. Ultrasonography revealed the intussusception was present in a long segment, and the patient was expected to have severe bowel congestion. We performed a laparoscopic exploration to find the location of the lesion and went through transumbilical single incision laparoscopic-assisted surgery to perform minimally invasive surgery. Exploration revealed the intussusception in the distal ileum, and we attempted intracorporeal laparoscopic reduction, but failed (Figure 2A). Therefore, a manual reduction was performed by pulling the proximal bowel via the navel window (Figure 2B). Although severe congestion of the small bowel was observed, there was no necrosis in the bowel. After replacing the bowel in the abdominal cavity, the surgery was completed (Figure 2C). The patient started a diet on the $3^{\text {rd }}$ postoperative day, and also started treatment for Henoch-schonlein purpura. The patient was discharged on the $9^{\text {th }}$ postoperative day with no further postoperative complications. Reduction of the other 5 ileocecal intussusceptions without leading points was very easy with transumbilical single incision laparoscopic-assisted surgeries with mean operation time $40 \pm 10$ minutes. In another case, a female patient aged 4.7 months had massive hematochezia and underwent a Meckel scan, which revealed a suspected lesion. Laparoscopic exploration was performed by transumbilical single incision laparoscopic-assisted surgery, and we discovered a $1 \mathrm{~cm}$ sized whitish, solid mass on the mesenteric border of a small bowel. Extracorporeal small bowel resection and anastomosis were performed through the port site. The lesion was confirmed to be a heterotrophic gastric mucosa (Figure 3). An intracorporeal band lysis was performed in 1 patient who was detected with an adhesion band that was suspected to occur after a traffic accident which resulted in partial bowel obstruction. The median age of patients who underwent appendectomy (72 patients) was 108 months (range: 32 months to 201 months) and mean operation time
Table 1. Patients Demographics $(\mathrm{n}=82)$

\begin{tabular}{|l|l|}
\hline Characteristics & Value \\
\hline Age (median, range) & $8.9 \mathrm{yrs}, 4.3 \mathrm{~m} \sim 16.7 \mathrm{yrs}$ \\
\hline Diagnosis & No. \\
\hline Acute appendicitis & $72(1$ ectopic pancreas) \\
\hline Intussusception & $6(5$ ileo-colic, 1 ileo-ileal:HSP*) \\
\hline Heterotrophic gastric mucosa & 1 \\
\hline Meckel's diverticulum c ectopic pancreas & 1 \\
\hline Intestinal obstruction & 1 (post-traumatic adhesion) \\
\hline Intestinal bleeding & 1 (transverse colon ulceration) \\
\hline
\end{tabular}

HSP: Henoch-schonleing purpura

for the procedure was $40 \pm 20$ minutes in appendectomy, whereas other surgeries ranged from 30 to 80 minutes (Table 2). The median length of hospital stay was 3 days ( 2 to 7 days) in appendectomy, and for other patients it ranged from 3 to 9 days. The patient discharged on the $9^{\text {th }}$ postoperative day had to stay longer because of the treatment for Henoch-schonlein purpura (Table 3). There were no immediate postoperative complications related to transumbilical single incision laparoscopic-assisted surgery. There were 3 cases with a stitch abscess which occurred about a month after the operation in appendectomy patients, and they were resolved after removing the stitch. Another patient visited complaining of an umbilical pain which spontaneously resolved with no other intervention. Umbilical scars were aesthetically superb (Figure 4).

\section{Discussion}

Due to the advancement of minimally invasive surgery, operative techniques are developing rapidly. The author always judges if the patient can be operated with minimally invasive surgery. In certain situations, minimally invasive surgery offers a better visualization than laparotomy. The benefit of minimally invasive surgery is accepted worldwide by surgeons, and numerous literature on laparoscopy and robot-assisted surgery has been published. Single-incision laparoscopic appendectomy in pediatric surgery was first introduced in the late 1990s [3,4]. Recently, a literature reporting bowel resection with a laparoscopic assisted transumbilical single incision port in a pediatric patient with Crohn's disease was published. Also published is a study comparing transumbilical laparoscopic-assisted appendectomy (TULA) and single incision laparoscopic appendectomy $[5,6]$. In 2015, Yuri, et al. reported reviewing single incision surgery in a pediatric patient and proposed the nomenclature 'single incision pediatric endosurgery' (SIPES). In the literature, they reported SIPES applied to neonates and patients under 12 months of age [2,7-9]. Numerous studies have address the feasibility of single incisions. As we noted, literature regarding the so called 'Hybrid operation' has also been introduced [10-12]. Technically, there are some differences 


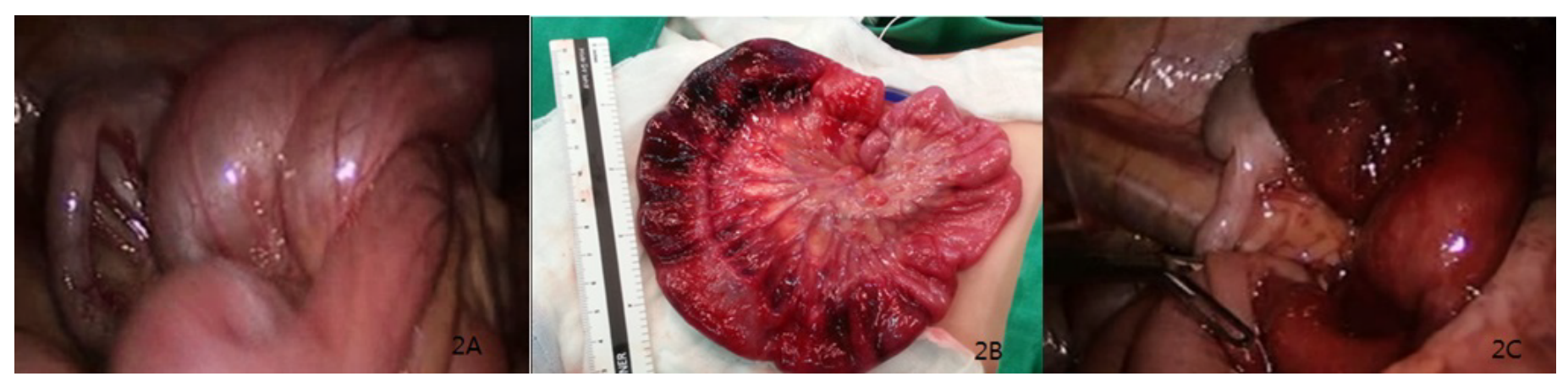

Figure 2. A) Ileo-ileal intussusception, B) Extracorporeal manual reduction, C) The appearance of the ileum that was returned to the abdomen after reduction

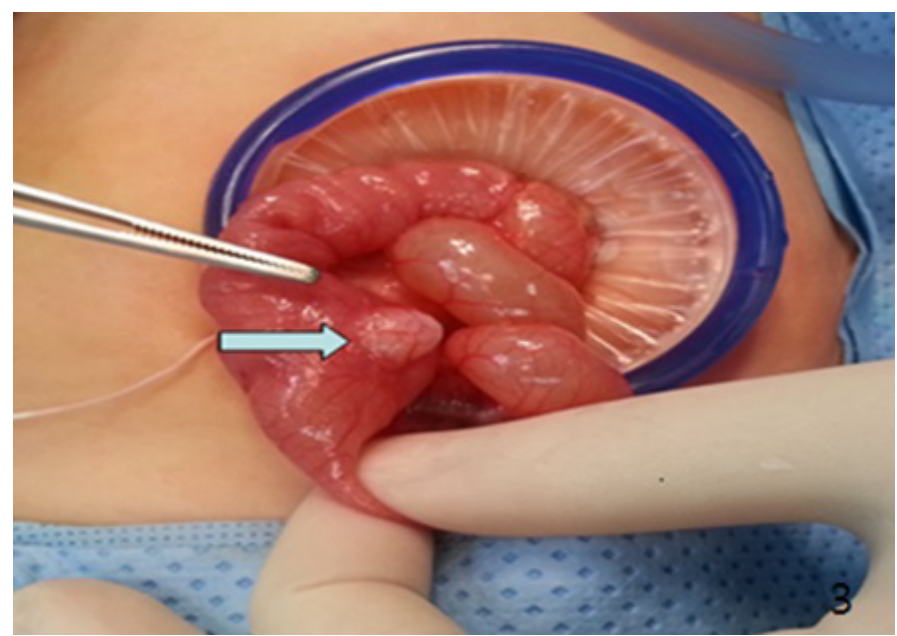

Figure 3. Heterotropic gastric mucosa in 4.7 months female patient

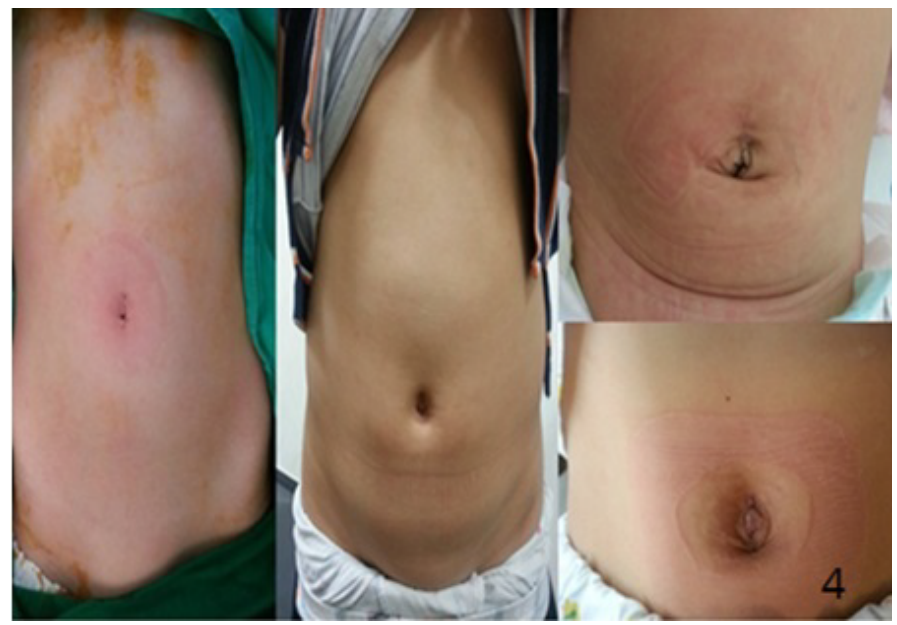

Figure 4. Postoperative umbilical scar

between when the transumbilical port is used only for laparoscopic procedure, and when the port is used for the extracorporeal procedure. Especially in intussusception, transumbilical single incision surgery was much more convenient than multi-port conventional laparoscopy. Conventional laparoscopic surgery to reduce intussusception is not easy. Surgery that pulls the congested intestine with the laparoscopic instrument is not only difficult because of the serosa tearing, but also requires an operation time of an hour or more to enable the intestine to return to its original position. However, by pulling out the proximal bowel through the umbilical window of the single port by hand, the reduction is completed very quickly. Even when the congestion is
Table 2. Transumbilcal single incision laparoscopic assisted surgery $(\mathrm{n}=82)$

\begin{tabular}{|c|c|c|}
\hline Diagnosis (n) & OP name & Op time (min) \\
\hline \multirow[b]{2}{*}{ Acute appendicitis (72) } & Appendectomy & $40 \pm 20$ \\
\hline & $\begin{array}{l}\text { Incidental small bowel } \\
\text { resection }\end{array}$ & 70 \\
\hline Intussusception (6) & $\begin{array}{l}\text { Manual reduction c pulling } \\
\text { method }\end{array}$ & $40 \pm 10$ \\
\hline $\begin{array}{l}\text { Heterotrophic gastric } \\
\text { mucosa (1) }\end{array}$ & $\begin{array}{l}\text { Small bowel resection \& } \\
\text { anastomosis }\end{array}$ & 60 \\
\hline $\begin{array}{l}\text { Meckel's diverticulum and } \\
\text { ectopic pancreas (1) }\end{array}$ & $\begin{array}{l}\text { Small bowel resection \& } \\
\text { anastomosis }\end{array}$ & 70 \\
\hline Intestinal obstruction (1) & Adhesiolysis & 50 \\
\hline Intestinal bleeding (1) & Exploration and adhesiolysis & 80 \\
\hline
\end{tabular}

Table 3. Length of hospital stay

\begin{tabular}{|l|c|c|}
\hline \multirow{2}{*}{ Diagnosis (n) } & \multicolumn{2}{|c|}{ Hospital days } \\
\cline { 2 - 3 } & Median & range \\
\hline Acute appendicitis (72) & 3 & $2 \sim 7$ \\
\hline Intussusception (2) & 7 & $3 \sim 9$ \\
\hline Heterotrophic gastric mucosa (1) & 5 & \\
\hline Meckel's diverticulum and ectopic pancreas (1) & 5 & \\
\hline Intestinal obstruction (1) & 5 & \\
\hline Intestinal bleeding (1) & 8 & \\
\hline
\end{tabular}

very severe, it usually does not exceed 30 minutes. Of the patients not included in this study period, 2 were not reduced by this method. Therefore, the open conversion was performed, and the duplication cysts were confirmed as the leading point in these 2 patients. In this method, the operation time for reduction of intussusception is faster than the conventional laparotomy; we recently performed the pulling method with transumbilical single incision laparoscopicassisted surgery in intussusception patients who are unsuccessful in air reduction. The limited intra-abdominal space in pediatric patients can be advantageous for utilizing the umbilical port that helps to reach more extensively than adult patients. That is why all single incision surgeries should be based on the hybrid operation. This assumption can also be altered for adult patients, especially in small bowel lesions like Meckel's diverticulum. Despite the fact that the concept of single incision laparoscopic-assisted surgery is not new, it is not widely applied in the field of pediatric surgery. Therefore, here we introduce our experience of transumbilical single incision laparoscopic-assisted surgery, which we consider to be superior to either laparotomy or conventional laparoscopy. The strength of this study is that we adjusted the transumbilical single incision laparoscopic-assisted surgery in intussusception. Although there were only 6 patients during the study period, the pulling methods with transumbilical single incision laparoscopic-assisted surgery were very easy and comfortable. In order to confirm this procedure, more research is required on a larger number of patients. In conclusion, transumbilical single incision 
laparoscopic-assisted surgery was applicable to appendectomy as well as various other surgical abdomen diseases in children. In particular, transumbilical single incision laparoscopic-assisted surgery may be a substitute for laparotomy in children who are suspected to require manipulation of the small bowel.

\section{Conflict of interest}

The authors declare that they have no conflict of interest.

\section{References}

1. Pelosi MA, Pelosi MA, (1992) Laparoscopic appendectomy using a single umbilical puncture (minilaparoscopy). J Reprod Med 37: 588-594.

2. Kozlov Y, Novozhilov V, Baradieva P, Krasnov P, Kovalkov K, et al. (2015) Singleincision pediatric endosurgery in newborns and infants. World J Clin Pediatr 4: 55-65.

3. Esposito C (1998) One-trocar appendectomy in pediatric surgery. Surg Endosc 12: 177 178. [Crossref]

4. Valla J, Ordorica-Flores RM, Steyaert H, Merrot T, Bartels A, et al. (1999) Umbilical one-puncture laparoscopic-assisted appendectomy in children. Surg Endosc 13: 83-85.
5. Boo YJ, Lee Y, Lee JS (2016) Comparison of transumbilical laparoscopic-assisted appendectomy versus single incision laparoscopic appendectomy in children: which is the better surgical option? J Pediatr Surg 51: 1288-1291. [Crossref]

6. Huntington JT, Boomer LA, Pepper VK, Diefenbach KA, Dotson JL, et al. (2016) Single-incision laparoscopic surgery (SILS) for children with Crohn's disease. Pediatr Surg Int 32: 459-464.

7. Takehara H, Yakabe S, Kameoka K (2006) Laparoscopic percutaneous extraperitoneal closure for inguinal hernia in children: clinical outcome of 972 repairs done in 3 pediatric surgical institutions. J Pediatr Surg 41: 1999-2003.

8. Muensterer OJ, Chong AJ, Georgeson KE, Harmon CM (2011) The Cross-technique for single-incision pediatric endosurgical pyloromyotomy. Surg Endosc 25: 3414-3418.

9. Clark JM, Koontz CS, Smith LA, Kelley JE (2008) Video-assisted transumbilica Meckel's diverticulectomy in children. Am Surg 74: 327-329. [Crossref]

10. Potter DD, Tung J, Faubion WA, Jr, Moir C (2012) Single-incision laparoscopic colon and rectal surgery for pediatric inflammatory bowel disease and polyposis syndromes. J Laparoendosc Adv Surg Tech A 22: 203-207.

11. Muensterer OJ, Keijzer R (2011) Single-incision pediatric endosurgery-assisted ileocecectomy for resection of a NEC stricture. Pediatr Surg Int 27: 1351-1353.

12. Hansen EN, Muensterer OJ, Georgeson KE, Harmon CM (2011) Single-incision pediatric endosurgery: lessons learned from our first 224 laparoendoscopic single-site procedures in children. Pediatr Surg Int 27: 643-648.

Copyright: $(02018$ Soo-Min J. This is an open-access article distributed under the terms of the Creative Commons Attribution License, which permits unrestricted use, distribution, and reproduction in any medium, provided the original author and source are credited. 